\title{
Development of new high-performance induction heating systems using model predictive control $^{1}$
}

\author{
S. Lucia ${ }^{\mathrm{a}}$, D. Navarro ${ }^{\mathrm{b}}$, H. Sarnago ${ }^{\mathrm{b}}$ and O. Lucia ${ }^{\mathrm{b}, *}$ \\ ${ }^{\mathrm{a}}$ TU Berlin, Einstein Center Digital Future, Einsteinufer 17, Berlin, Germany. \\ ${ }^{\mathrm{b}}$ University of Zaragoza, Maria de Luna, 1, Zaragoza, Spain
}

\begin{abstract}
Modern induction heating systems involve usually many non-linear systems with different constraints, making difficult implementing classical controllers. In this context, model predictive control arises as a powerful technique able to provide high performance controllers with advanced functionalities. This paper will discuss the benefits of this control technique for induction heating systems and will provide an example of application for domestic systems. As a conclusion, MPC will help powering future induction heating systems with improved control capabilities and performance.
\end{abstract}

Keywords: Induction heating, model predictive control, optimization, power electronics, resonant power conversion

\section{Introduction}

Induction heating consist on applying an alternating magnetic field to a material, usually with ferromagnetic properties, to induce currents that will lead to the material heating. Induction heating (IH) has spread to a number of applications [1] ranging from industrial to biomedical or domestic ones due to its benefits in terms of performance and efficiency. Among the main benefits of IH, fast heating, repetitive and accurate process, safety and being a contactless heating method are the most relevant ones.

IH systems usually rely on a power converter in order to generate the required high frequency current/voltage to supply the coil that generates the alternating magnetic field (Fig. 1). These systems usually feature resonant power converters [2] to obtain the required medium frequency currents, which are sometimes combined with $\mathrm{dc}-\mathrm{dc}$ converters for better load-power adaptation and power factor correction [3]. Modern IH systems are highly non-linear and, consequently, designing the control scheme requires taking into account a wide variety of constraints including power converter, heating process, and industrial process constraints. Under these conditions, classic control techniques are difficult to tune and include all the possible constraints and desired performance.

In the past, several approaches have been followed to try to achieve high-performance controllers. As the load characteristics changes with temperature, usually the controller task becomes heavy. An original

\footnotetext{
${ }^{*}$ Corresponding author: O. Lucia, University of Zaragoza, Maria de Luna, 1, 50018 Zaragoza, Spain. Tel.: +34 876555319; E-mail: olucia@unizar.es.

${ }^{1}$ An earlier version of this paper was presented during the Heating by Electromagnetic Sources HES19 conference.
} 


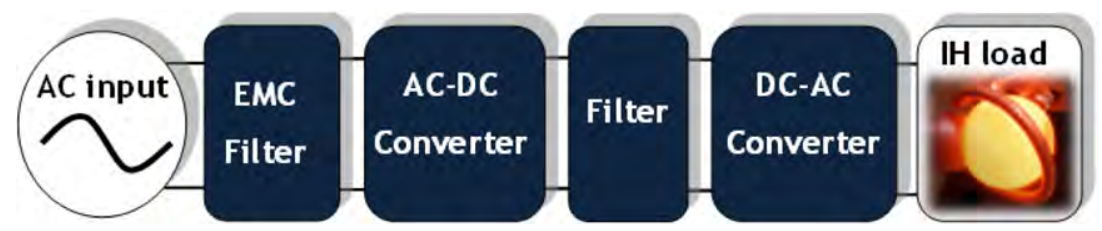

Fig. 1. Typical power conversion flow in an IH system.

control scheme is described and tested in [4-6] for a six-coil system called zone-controlled induction heating $(\mathrm{ZCIH})$ in a longitudinal flux configuration. Paper [6] presents the use of a current control method based on a circuit model using real and imaginary current/voltage components instead of the current amplitude and phase angle control in the classical approaches. State feedback decoupling provides separate current control on each phase with high dynamics but with a high computation burden due to numerous high rank matrix transformations. The six currents remain in phase in this application where no dcdc converter is needed unlike in [4]. In order to obtain appropriate dynamics, resonant control of the inductor currents has been presented in [7] versus other classical control techniques. However, still all these techniques are based on linearized control techniques that makes difficult, or not feasible at all, the use of complex constraints and control targets.

All these modulation and control algorithms have been implemented either using analog [8] or digital techniques. Nowadays, digital implementations using digital signal processors (DSPs) or fieldprogrammable gate arrays (FPGAs) are preferred due to their advantages in terms of configurability and performance. In particular, several design methodologies [9], monitoring [10] and control architectures [11-13], and accelerated simulation techniques [14] for IH systems have been proposed taken advantage of FPGAs. Still, real-time implementation of advanced controllers using cost-effective digital control hardware is a challenge.

In this context, model predictive control (MPC) arises as a powerful tool to provide high-performance controllers being able to include different nature restrictions in the optimization process. This paper will review the fundamentals of model predietive control and its applications and will provide an application example and future perspectives.

\section{Fundamentals of model predictive control}

Model predictive control uses the mathematical model of a dynamic system to predict its future behaviour and compute a sequence of optimal control inputs to satisfy given constraints and optimize a desired performance measure (Fig. 2).

The mathematical model used is usually described in the form of differential equations as $\dot{x}=f(x, u, p)$, where $x$ are the states of a system, $u$ the available control inputs and $p$ different parameters that affect the dynamics. MPC solves an optimization to problem to compute the optimal trajectory of control inputs up to a certain point into the future, called the prediction horizon. The first part of the optimal trajectory is applied to the system and at the next sampling time, the same procedure is repeated. The optimization problem solved at each sampling time can be written as

$$
\underset{u(\cdot)}{\operatorname{minimize}} \int_{t=0}^{t_{\text {end }}} J(x(t), u(t), p) d t
$$

subject to $\dot{x}=f(x, u, p), g(x, u, p) \leq 0$ 


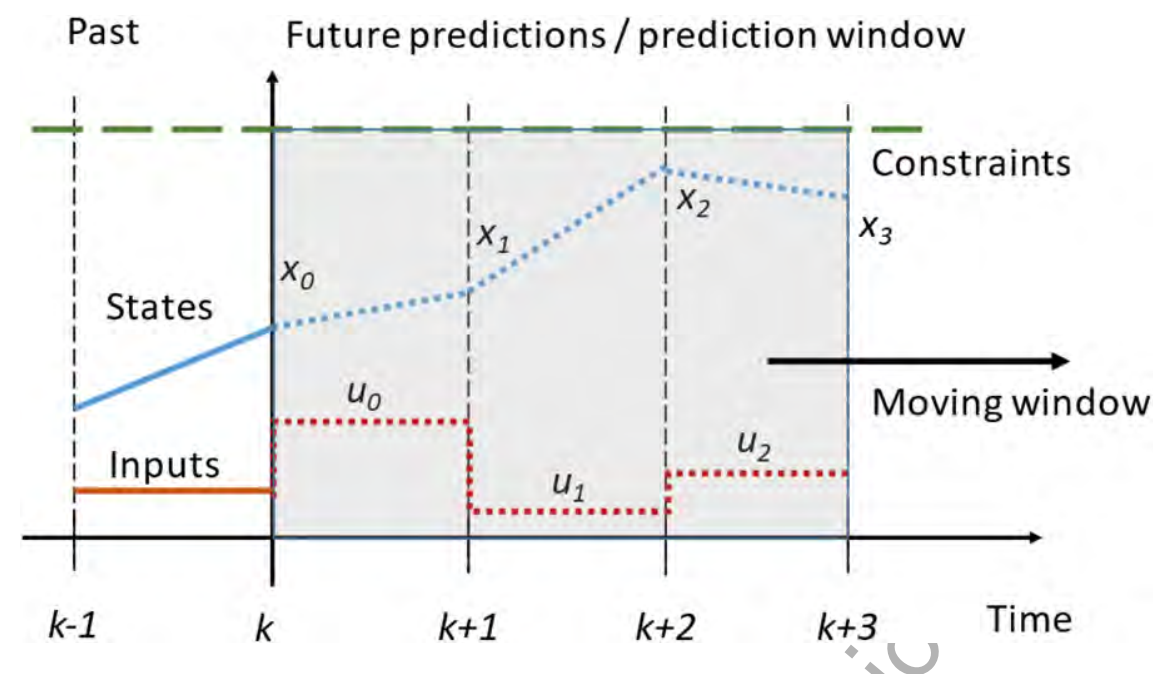

Fig. 2. Schematic description of Model Predictive Control. A mathematical model is used to predict the future states of a dynamic system and compute a sequence of optimal control inputs by solving an optimization problem.

where $J(\cdot)$ denotes the objective function (desired control objective). The constraints of the optimization problem are the system dynamics and any other operational constraint denoted by $g(\cdot)$. The flexibility in the choice of the performance goal and constraints makes MPC a very powerful control technique that can be applied in a very large range of applications. To facilitate the numerical solution, usually a piecewise parametrization of the input signal is performed, as shown in Fig. 2, and the differential equations and integral of the objective function are also discretized. Solving the optimization problem in real time used to be the main challenge of MPC. However, recent advances in algorithms, hardware and tailored implementations have enabled the possibility of implementing MPC controllers for fast systems, including also power electronics applications.

\section{MPC for induction heating systems}

In the past, MPC has been applied to many industrial systems, from aerospace and mechanical systems, to chemical processes. In the last years, MPC has been also applied to electrical/electronic systems, providing a breakthrough in control possibilities in modern power electronic systems [15]. These control approaches have opened the window for improved performance in many areas.

However, considering IH applications, little research or industrial applications have been yet described. In [16], a furnace control is proposed and compared with current state-of-the-art alternatives, highlighting benefits of MPC, and an IH furnace with a parallel-resonant load and unity input power factor is described in [17]. In [18], Laguerre functions are applied in order to obtain a less demanding computational complexity.

Despite its little current adoption, there is a great potential for MPC applications to IH due to several distinctive elements inherent of IH processes. Some of them are summarized as follows:

Temperature evolution. IH processes, by definition, produce changes in the temperature of the IH target that may be very fast and in a wide range. These may lead to changes in the plant to control, becoming a challenging control problem. MPC can therefore provide tools to identify temperature variation, predict its evolution and, consequently, optimize the controller. 
Geometry and coupling. Geometry of the IH system and, specifically, the electromagnetic coupling of the IH coil and the IH target are challenging aspects. This may significantly change the plant and, consequently, lead to different performances. This is especially important in those systems with a wide load variation, such as domestic induction heating, where a wide variety of geometries and positions are used in a non-predictable way. In this context, MPC again provides tools to predict and adapt the controller to these plant variations.

Resonant tank and soft-switching. Usually, power converters for induction heating systems rely on the use of resonant converters [2] to achieve high-efficiency [19]. Despite their benefits, resonant power converters require fine tuning of the resonant tank and the soft-switching conditions. In this context, MPC is a valuable ally in order to ensure that the power converter is being operated in a high-efficiency and reliable operating point being able to consider complex constraints.

Component dispersion and aging. Finally, as in any other electronic system, there can be a significant effect of the component dispersion due to tolerances or aging in the final plant performance. This is especially true in IH systems which suffer usually heavy thermo-mechanical stress, use capacitive and magnetic elements with wide tolerances and geometrical variations, and rely on resonant converters highly affected by these effects. For these reasons, MPC is a helpful ally to cope with these elements and to optimize the controller under this wide range of operating conditions.

The MPC implementation consist on defining a function to be optimized subject to a certain set of constraints. By putting this formulation in the context of $\mathrm{IH}$, a set of parameters is discussed as follows.

When dealing with the optimization function, different options are possible depending upon the application. The most obvious is the target output power, which is the desired target in many industrial IH processes. However, the actual target temperature can be also considered, either by direct measurement or by using real-time estimators. Other possibilities such as the system efficiency, switching frequency or settling time can also be considered. Moreover, the combination of several of these factors can be considered by using weighting factors in the optimization function, making possible to achieve complex performance targets in a straight-forward way not possible using conventional control methods.

The constraints will be taken into account to obtain the optimum controller in MPC. Unlike other classical control approaches, complex constraints such as instantaneous magnitude values, ranges, or Boolean conditions can be considered. Assuming the IH context, important parameters such as current and voltage, efficiency, switching frequency ranges, power and/or temperature limits or soft-switching conditions can be considered. This' opens the windows to a far wider range of optimization possibilities, making possible to implement higher performance systems, with higher efficiency and reliability. Besides, since the performance can be optimized and the stress on each subsystem part can be optimized, costeffective implementations can be achieved.

Some of these benefits and formulation examples are discussed in the next Section, where an application example to domestic induction heating is presented.

\section{Application example: Domestic IH}

Domestic induction heating [20] (Fig. 3) is a representative example of IH system [1] where the IH target is a pan/pot for culinary purposes [21]. It has many elements in common with industrial or biomedical systems, and some specific challenges, especially regarding the wide output power and load coupling variability [22]. In this Section, an example of application of MPC to domestic IH $[23,24]$ is briefly discussed. 


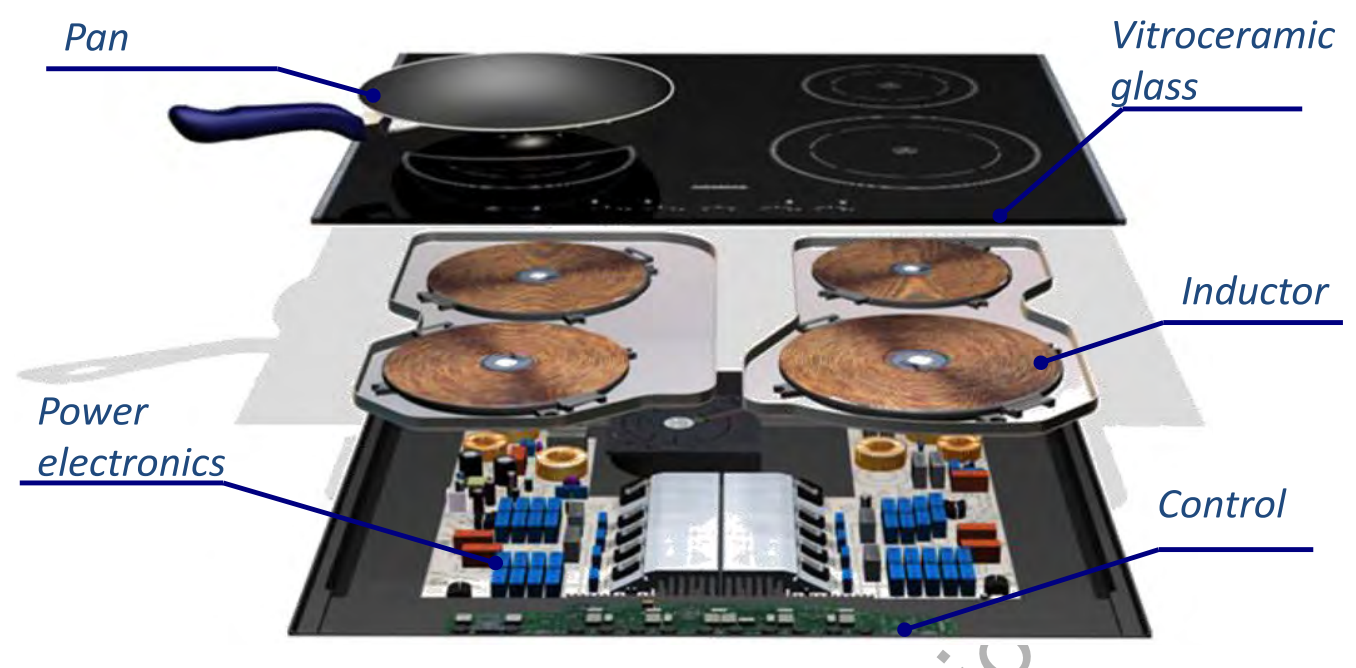

Fig. 3. Induction heating home appliance.

Domestic induction heating is based on creating an alternating magnetic field to generate heat in the induction target by means of Eddy currents and magnetic hysteresis [1]. Typically, planar induction coils to fit the low-profile structure are used (see Fig. 3), and resonant power conversion as discussed before is applied.

Domestic IH requires optimizing the power converter operation, i.e. soft-switching and reduced switching frequency for high efficiency, while avoiding acoustic noise. This is especially important in this application, where the user performance is defined by the perceived output power and noise. Consequently, the constraints discussed in the previous section severely affects to this application.

This problem considers a half-bridge series resonant inverter (Fig. 4) that is controlled using both switching frequency $f_{s w}$ and duty cycle $D$. In this example (see expression (2)), the cost function (a) considers both the output power $P_{o}$ and the switching frequency $f_{s w}$, weighted by the parameter $\alpha$. By doing this, the required output power is obtained while the switching frequency is minimized in order to minimize switching losses. This optimization is evaluated each switching period, providing optimized transient performance.

$$
\begin{array}{cl}
\underset{f_{s w, k}, D_{k}}{\operatorname{minimize}} & \sum_{k=0}^{N}\left(P_{o, k}-P_{o}^{d e s}\right)^{2}+\alpha f_{s w, k} \\
\text { subject to } & \text { dynamic model } \\
& 30 \leq f_{s w, k} \leq 100 \mathrm{kHz}, \\
& 0.2 \leq D_{k} \leq 0.8, \\
& i_{o, k} \geq 0, \text { if } k=1,3,5, \ldots, N-1, \\
& i_{o, k} \leq 0, \text { if } k=0,2,4, \ldots, N, \\
& D_{k}=D_{k-1}, \text { if } k=1,3,5, \ldots, N-1, \\
& f_{s w, k}=f_{s w, k-1}, \text { if } k=1,3,5, \ldots, N-1
\end{array}
$$

Several parameters are established as key constraints. First of all, since there are multiple combinations of $f_{s w}$ and $D$ to obtain the same output power, several thresholds are defined to avoid acoustic noise and limit maximum frequency (c), and to limit current distortion (d). Besides, in order to ensure zero voltage 


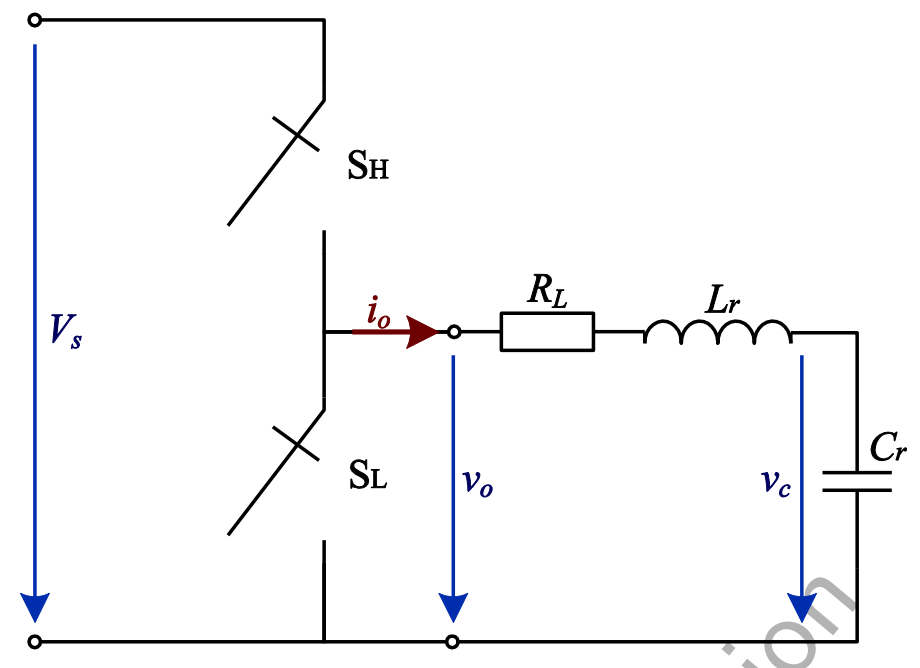

Fig. 4. Half-bridge series resonant converter.

switching (ZVS) operation, i.e. soft-switching, the sign of the current is checked in constraints (e) and (f) after each semi-cycle. It is important to note that this controller designs leads to a high-efficiency and reliable operation, and these considerations cannot be taken into account using classical linear controllers.

Figure 5 shows the simulation results obtained by solving problem (2) after each switching cycle for a conventional domestic IH load, including the main waveforms, control inputs, and output power. In this figure, it can be seen that the controller achieves the target output power (dashed line in the bottom plot) within a few switching cycles while keeping the control inputs within the desired ranges. Moreover, the controller ensures that ZVS operation is achieved, even during transient behaviour, by ensuring the proper current sign during the switching times, guaranteeing consequently a high-efficiency and reliable operation. As a conclusion, MPC has proved to be a useful tool for improving control systems of domestic IH systems subject to a wide variety of constraints [25].

\section{Future perspectives and conclusions}

Domestic induction heating technology is gaining acceptance in the domestic market due to its benefits in terms of performance, efficiency and safety. The development of this technology and its modern applications requires new and innovative power converter design with increasingly complex control strategies. These control strategies must take into account not only the output power target, but also complex constraints such as soft-switching operation, acoustic and flicker emissions, and switching frequency constraints, among others. In this context, classical control techniques cannot provide an easy implementation to fit all these requirements.

MPC is a powerful tool to implement modern high-performance IH controllers, where complex constraints can be considered in the optimization process. This paper has reviewed the state-of-the-art and main applications, and has detailed an example of application for domestic IH. This example has proved the ability of MPC to ensure, not only the output power requirements, but also complex operational conditions optimization in a feasible manner. Future challenges will include taking into account additional restrictions as well as the embedded implementation of these controllers [26]. 


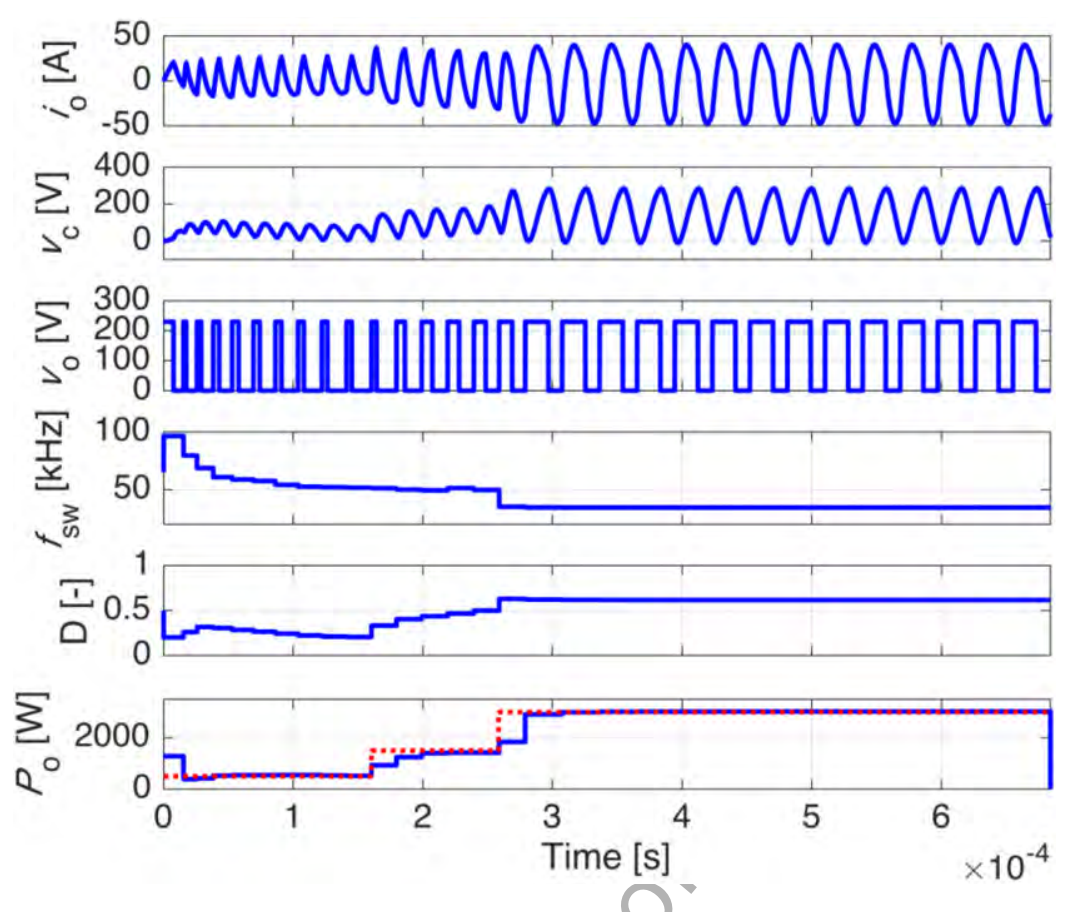

Fig. 5. MPC applied to domestic IHperformance results [24].

\section{Acknowledgements}

This work was partly supported by the Spanish MINECO under Project TEC2016-78358-R, by the Spanish MICINN and AEI under Project RTC-2017-5965-6, co-funded by EU through FEDER program, by the DGA-FSE, and by the BSH Home Appliances Group.

\section{References}

[1] O. Lucía, P. Maussion, E. Dede and J.M. Burdío, Induction heating technology and its applications: Past developments, current technology, and future challenges, IEEE Transactions on Industrial Electronics 61 (2014), 2509-2520, doi:10.1109/TIE.2013.2281162.

[2] H. Sarnago, O. Lucía, A. Mediano and J.M. Burdío, Analytical model of the half-bridge series resonant inverter for improved power conversion efficiency and performance, IEEE Transactions on Power Electronics 30 (2015), 4128-4143, doi:10.1109/TPEL.2014.2359576.

[3] M. Pérez-Tarragona, H. Sarnago, O. Lucía and J.M. Burdío, Design and experimental analysis of PFC rectifiers for domestic induction heating applications, IEEE Transactions on Power Electronics 33 (2017), 6582-6594, doi:10.1109/TPEL.2017.2755367.

[4] H. Pham, H. Fujita, K. Ozaki and N. Uchida, Phase angle control of high-frequency resonant currents in a multiple inverter system for zone-control induction heating, IEEE Transactions on Power Electronics 26 (2011), 3357-3366, doi:10.1109/TPEL.2011.2146278.

[5] H.N. Pham, H. Fujita, K. Ozaki and N. Uchida, Estimating method of heat distribution using 3-D resistance matrix for zone-control induction heating systems, IEEE Transactions on Power Electronics 27 (2012), 3374-3382, doi:10.1109/tpel.2011.2179984.

[6] H.N. Pham, H. Fujita, K. Ozaki and N. Uchida, Dynamic analysis and control for resonant currents in a zone-control induction heating system, IEEE Transactions on Power Electronics 28 (2013), 1297-1307, doi:10.1109/tpel.2012.2210286. 
[7] L. Nguyen Kien, S. Caux, X. Kestelyn, O. Pateau and P. Maussion, Resonant control of multi-phase induction heating systems, in: Annual Conference on IEEE Industrial Electronics Society IECON, 2012, pp. 3293-3298.

[8] J.I. Artigas, I. Urriza, J. Acero, L.A. Barragán, D. Navarro and J.M. Burdío, Power measurement by outputcurrent integration in series resonant inverters, IEEE Transactions on Industrial Electronics 56 (2009), 559-567, doi:10.1109/TIE.2008.2003215.

[9] D. Navarro, O. Lucía, L.A. Barragán, I. Urriza and O. Jiménez, High-level synthesis for accelerating the FPGA implementation of computationally-demanding control algorithms for power converters, IEEE Transactions on Industrial Informatics 9 (2013), 1371-1379, doi:10.1109/TII.2013.2239302.

[10] O. Jiménez, O. Lucía, L.A. Barragán, D. Navarro, J.I. Artigas and I. Urriza, FPGA-based test-bench for resonant inverter load characterization, IEEE Transactions on Industrial Informatics 9 (2013), 1645-1654, doi:10.1109/TII.2012.2226184.

[11] O. Lucía, L.A. Barragán, J.M. Burdío, O. Jiménez, D. Navarro and I. Urriza, A versatile power electronics test-bench architecture applied to domestic induction heating, IEEE Transactions on Industrial Electronics 58 (2011), 998-1007, doi:10.1109/TIE.2010.2048840.

[12] D. Navarro, O. Lucía, L.A. Barragán, J.I. Artigas, I. Urriza and O. Jiménez, Synchronous FPGA-based implementations of digital pulse width modulators, IEEE Transactions on Power Electronics 27 (2012), 2515-2525, doi:10.1109/TPEL.2011.2173702.

[13] O. Jiménez, O. Lucia, I. Urriza, L.A. Barragán, P. Mattavelli and D. Boroyevich, An FPGA-based gain-scheduled controller for resonant converters applied to induction cooktops, IEEE Transactions on Power Electronics 29 (2014), 2143-2152, doi:10.1109/TPEL.2013.2276041.

[14] O. Lucía, I. Urriza, L.A. Barragan, D. Navarro, O. Jiménez and J.M. Burdío, Real-time FPGA-based hardware-in-the-loop simulation test-bench applied to multiple output power converters, IEEE Transactions on Industry Applications 47 (2011), 853-860, doi:10.1109/TIA.2010.2102997.

[15] S. Kouro, M.A. Perez, J. Rodriguez, A.M. Llor and H.A. Young, Model predictive control: MPC's role in the evolution of power electronics, IEEE Industrial Electronics Magazine 9 (2015), 8-21, doi:10.1109/MIE.2015.2478920.

[16] G.C. Goodwin, R.H. Middleton, M.M. Seron and B. Campos, Application of nonlinear model predictive control to an industrial induction heating furnace, Annual Reviews in Control 37 (2013), 271-277, doi:10.1016/j.arcontrol.2013.09.006.

[17] M. Nawaz, M.A. Saqib, S.A.R. Kashif and M. Gul, Constrained model predictive control for an induction heating load, Transactions of the Institute of Measurement and Control (2018), doi:10.1177/0142331218758887.

[18] M. Nawaz, M.A. Saqib, S.A.R. Kashif and Z.U.R. Ch, Orthonormal functions based model predictive control of a currentsource resonant inverter, in: 2017 International Conference on Electrical Engineering (ICEE), 2017, pp. 1-5.

[19] H. Sarnago, O. Lucía, A. Mediano and J.M. Burdío, Analysis and design of high-efficiency resonant inverters for domestic induction heating applications, International Journal of Applied Electromagnetics and Mechanics 44 (2014), 201-208, doi:10.3233/JAE-141760.

[20] O. Lucía, J. Acero, C. Carretero and J.M. Burdío, Induction heating appliances: Towards more flexible cooking surfaces, IEEE Industrial Electronics Magazine 7 (2013), 35-47, doi:10.1109/MIE.2013.2247795.

[21] L. Qiu et al., Study on improving heating efficiency of induction cooker system by using an adiabatic layer, (2018), 1-10. doi:10.3233/JAE-180072.

[22] O. Lucía, J.M. Burdío, I. Millán, J. Acero and D. Puyal, Load-adaptive control algorithm of half-bridge series resonant inverter for domestic induction heating, IEEE Transactions on Industrial Electronics 56 (2009), 3106-3116, doi:10.1109/TIE.2009.2022516.

[23] S. Lucia, D. Navarro, B. Karg, H. Sarnago and O. Lucia, Deep learning-based model predictive control for resonant power converters, arXiv e-prints (2018), arXiv:1810.04872v1.

[24] S. Lucía, D. Navarro, H. Sarnago and O. Lucía, Model predictive control for resonant power converters applied to induction heating, in: IEEE International Symposium on Industrial Electronics, 2018, pp. 246-251.

[25] M. Nakagawa and H. Yonemori, A study on the audible frequency area noise emanating from a pan when the IH cooker is fed by the power source including harmonics, 59 (2018), 1-10. doi:10.3233/JAE-171236.

[26] S. Lucia, D. Navarro, O. Lucia, P. Zometa and R. Findeisen, Optimized FPGA implementation of model predictive control for embedded systems using high level synthesis tool, IEEE Transactions on Industrial Informatics 14 (2017), 137-145, doi:10.1109/TII.2017.2719940. 